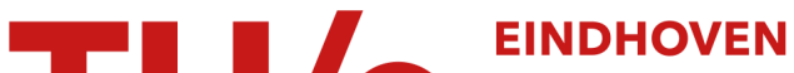 \\ UNIVERSITY OF \\ TECHNOLOGY
}

\section{Dynamics of two identical vortices in linear shear}

Citation for published version (APA):

Trieling, R. R., Dam, C. E. C., \& Heijst, van, G. J. F. (2010). Dynamics of two identical vortices in linear shear. Physics of Fluids, 22(11), 117104-1/13. [117104]. https://doi.org/10.1063/1.3489358

DOI:

10.1063/1.3489358

Document status and date:

Published: 01/01/2010

\section{Document Version:}

Publisher's PDF, also known as Version of Record (includes final page, issue and volume numbers)

\section{Please check the document version of this publication:}

- A submitted manuscript is the version of the article upon submission and before peer-review. There can be important differences between the submitted version and the official published version of record. People interested in the research are advised to contact the author for the final version of the publication, or visit the $\mathrm{DOI}$ to the publisher's website.

- The final author version and the galley proof are versions of the publication after peer review.

- The final published version features the final layout of the paper including the volume, issue and page numbers.

Link to publication

\section{General rights}

Copyright and moral rights for the publications made accessible in the public portal are retained by the authors and/or other copyright owners and it is a condition of accessing publications that users recognise and abide by the legal requirements associated with these rights.

- Users may download and print one copy of any publication from the public portal for the purpose of private study or research.

- You may not further distribute the material or use it for any profit-making activity or commercial gain

- You may freely distribute the URL identifying the publication in the public portal.

If the publication is distributed under the terms of Article 25fa of the Dutch Copyright Act, indicated by the "Taverne" license above, please follow below link for the End User Agreement:

www.tue.nl/taverne

Take down policy

If you believe that this document breaches copyright please contact us at:

openaccess@tue.nl

providing details and we will investigate your claim. 


\title{
Dynamics of two identical vortices in linear shear
}

\author{
R. R. Trieling, ${ }^{\text {a) }}$ C. E. C. Dam, and G. J. F. van Heijst \\ Fluid Dynamics Laboratory, Eindhoven University of Technology, P.O. Box 513, 5600 MB Eindhoven, \\ The Netherlands
}

(Received 10 February 2010; accepted 10 August 2010; published online 11 November 2010)

\begin{abstract}
The dynamics of two identical vortices in linear shear was studied both numerically and experimentally. Numerical simulations based on the technique of contour dynamics reveal that the vortex evolution in adverse shear is significantly different from that in cooperative shear. Vortices in adverse shear predominantly separate, whereas vortices in cooperative shear predominantly merge. In addition, adverse shear may destruct the vortices much in the same way as a single vortex in adverse shear, whereas cooperative shear stabilizes the vortices and thus enhances the possibility of vortex merger. The critical distance for vortex merger depends strongly on both the sign and the strength of the linear shear and, to a lesser extent, on the initial vorticity distribution. A simple vortex merger criterion is derived based on the interaction of two point vortices in linear shear. The different behavior of vortices in adverse and cooperative shear was confirmed by rotating-tank experiments. (C) 2010 American Institute of Physics. [doi:10.1063/1.3489358]
\end{abstract}

\section{INTRODUCTION}

One of the most striking features of two-dimensional (2D) turbulence is the development of coherent vortices from smaller-scale structures, a process generally referred to as "self-organization" (see, for example, Refs 1 and 2). Kinetic energy initially distributed over larger and smaller eddies eventually concentrates in large-scale structures that dominate the subsequent flow evolution. A solid understanding of the interactions of these vortices is therefore crucial in order to gain insight in 2D turbulence itself.

A fundamental process in $2 \mathrm{D}$ turbulent flows is the merger of two nearby like-signed vortices. For this reason, a number of studies have been devoted to the understanding of the key mechanisms of this process. ${ }^{3-8}$ Previous experimental and numerical studies ${ }^{3,9-15}$ have shown that two identical vortices merge if their initial intercentroid distance is smaller than some critical value, leading to a vortex larger in size than the original vortices. It was also shown that the interaction of unequal corotating vortices is much richer than that of equal vortices. ${ }^{16-20}$ Whereas the merger of two identical vortices produces only a single vortex, the close interaction of unequal vortices can often produce two vortices, one of which may be weaker than the original ones.

All these results are based on the interaction of vortices in isolation. In a turbulent flow, however, vortices are generally influenced by the shearing of surrounding vortices, which may significantly affect the onset to the vortex merger. Indeed, a nonuniform background velocity may push the vortices further apart or bring them closer together, so that it may either be easier or more difficult for vortices to merge in a background flow than without it. This situation is especially relevant for the initial and intermediate stages of decaying 2D turbulence in which vortices are relatively closely separated.

${ }^{a)}$ Electronic mail: r.r.trieling@tue.nl. URL: www.fluid.tue.nl.
As a first approximation, the effect of neighboring vortices may be represented by linear shear, being the first component of a local Taylor series expansion of the complex velocity field in which each vortex is embedded. Previous studies ${ }^{21-25}$ have shown that single vortices embedded in adverse and cooperative shear behave very differently. Vortices in adverse shear may be torn apart into a long thin filament due to the joint effect of vortex stripping and horizontal diffusion, whereas vortices in cooperative shear may evolve to equilibria that are nearly elliptical with their major axis aligned in the direction of the background shear. The different behavior of vortices in adverse and cooperative shear is also to be expected for the merger of two corotating vortices and may drastically affect the criteria for vortex merger.

In this paper, the interaction of two equal vortices in a linear shear flow is studied both numerically and experimentally. As a first approach, the vortices are modeled by point vortices, whereas the linear shear is imposed analytically. The point-vortex model enables us to concentrate on the main dynamical aspects of the evolution and offers the simplest possible configuration to quantify. For identical point vortices, the problem can be described with only a single dimensionless parameter. The point-vortex model gives important insight in the different behavior of vortices in adverse and cooperative shear. As a second approach, we apply the technique of contour dynamics, ${ }^{26,27}$ which is based on the twin requirement that the vorticity is both materially conserved and piecewise uniform. This technique allows us to investigate the effect of distributed vorticity on the interaction of vortices in linear shear.

The experiments are carried out in a rotating tank in which the flow is quasi-two-dimensional. Similar techniques are used as in previous studies ${ }^{25,28,29}$ to generate the vortices and the linear shear. The vortices are created by locally siphoning fluid through perforated tubes for a short time period, whereas the linear shear is created by a sudden increase in the angular velocity of the tank. A number of experiments 


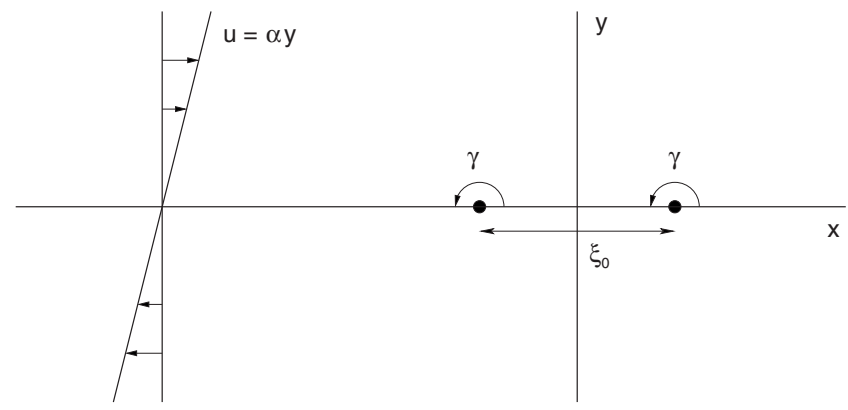

FIG. 1. Initial configuration of two point vortices, each with circulation $\gamma$, located an initial distance $\xi_{0}$ apart in linear shear with strength $\alpha$.

are performed with different values for the shear strength and the intercentroid distance. The subsequent flow evolution is visualized by injecting different colors of dye into the cores of the initial vortices, whereas particle-tracking techniques provide quantitative information about the horizontal flow field. Both the numerical and experimental results are summarized in regime diagrams. Special attention is given to the differences between the evolutions in adverse and cooperative shear.

This paper is organized as follows. Section II gives a description of the point-vortex model. Next, in Sec. III, the contour dynamics method is introduced and the results for two types of vortices with distributed vorticity are discussed. Section IV discusses the results of the laboratory experiments. Finally, in Sec. V, the results are summarized and the conclusions are given.

\section{POINT-VORTEX MODEL}

In order to explain the key dynamical aspects of the flow evolution, we consider two point vortices, each with strength $\gamma$, located an initial distance $\xi_{0}$ apart. The point vortices are initially aligned in the flow direction of a linear shear flow with strength $\alpha$, whose velocity components in the $x$ - and $y$-directions are given by $u=\alpha y$ and $v=0$, respectively (see Fig. 1). The time evolution of this two-vortex system depends on three parameters: the initial distance between the point vortices $\xi_{0}$, the strength of each point vortex $\gamma$, and the strength of the shear flow $\alpha$. On dimensional grounds, a single dimensionless parameter can be defined which characterizes the entire flow evolution

$$
\mu=\frac{\alpha \xi_{0}^{2}}{\gamma}
$$

When $\mu<0$, the shear is referred to as cooperative; when $\mu>0$, the shear is called adverse.

The motion of two identical point vortices in a linear shear flow has been studied before by Kimura and Hasimoto $^{30}$ and also by Waugh, ${ }^{31}$ but with a different orientation of the axes and for adverse shear only. Below we will closely follow the analysis of Kimura and Hasimoto.

The equations of motion for two point vortices of strength $\gamma$ in a linear shear flow of strength $\alpha$ are given by

$$
\frac{d x_{1}}{d t}=-\frac{\gamma}{2 \pi} \frac{y_{1}-y_{2}}{r^{2}}+\alpha y_{1},
$$

$$
\begin{aligned}
& \frac{d y_{1}}{d t}=\frac{\gamma}{2 \pi} \frac{x_{1}-x_{2}}{r^{2}}, \\
& \frac{d x_{2}}{d t}=-\frac{\gamma}{2 \pi} \frac{y_{2}-y_{1}}{r^{2}}+\alpha y_{2}, \\
& \frac{d y_{2}}{d t}=\frac{\gamma}{2 \pi} \frac{x_{2}-x_{1}}{r^{2}}
\end{aligned}
$$

where $\left(x_{i}, y_{i}\right)$ is the position of the $i$ th vortex and $r=\sqrt{\left(x_{1}-x_{2}\right)^{2}+\left(y_{1}-y_{2}\right)^{2}}$ is the distance between the vortices. This system of two interacting point vortices in linear shear is a Hamiltonian dynamical system, so that the equations of motion can be written in the forms

$$
\begin{aligned}
& \frac{\partial H}{\partial y_{i}}=\gamma \frac{d x_{i}}{d t}, \\
& -\frac{\partial H}{\partial x_{i}}=\gamma \frac{d y_{i}}{d t},
\end{aligned}
$$

where

$$
H=-\frac{\gamma^{2}}{4 \pi} \ln \left\{\left(x_{1}-x_{2}\right)^{2}+\left(y_{1}-y_{2}\right)^{2}\right\}+\frac{\alpha \gamma}{2}\left(y_{1}^{2}+y_{2}^{2}\right)
$$

is the Hamiltonian of the system.

Equations (3) and (5) yield an integral quantity

$$
Y=\left(y_{1}+y_{2}\right) / / 2,
$$

which is the $y$-component of the center of vorticity of the two-vortex system. The corresponding $x$-component is given by

$$
X=\left(x_{1}+x_{2}\right) / 2 .
$$

Using Eqs. (2)-(5), the time dependence of the center of vorticity is found to be

$$
\begin{aligned}
& X(t)=\alpha Y_{0} t+X_{0}, \\
& Y(t)=Y_{0},
\end{aligned}
$$

where $X_{0}$ and $Y_{0}$ are the initial values of $X$ and $Y$. Thus it follows that the center of vorticity moves along with the local velocity of the shear flow.

Introducing the relative coordinates

$$
\begin{aligned}
& \xi=x_{2}-x_{1}, \\
& \eta=y_{2}-y_{1},
\end{aligned}
$$

the Hamiltonian can be rewritten as

$$
H=-\frac{\gamma^{2}}{4 \pi} \ln \left(\xi^{2}+\eta^{2}\right)+\frac{\alpha \gamma}{2} \frac{1}{2}\left[\eta^{2}+\left(2 Y_{0}\right)^{2}\right] .
$$

Noting that the Hamiltonian of the system is a constant of motion, an analytical expression for the trajectories of the vortices can be derived, which is given by 


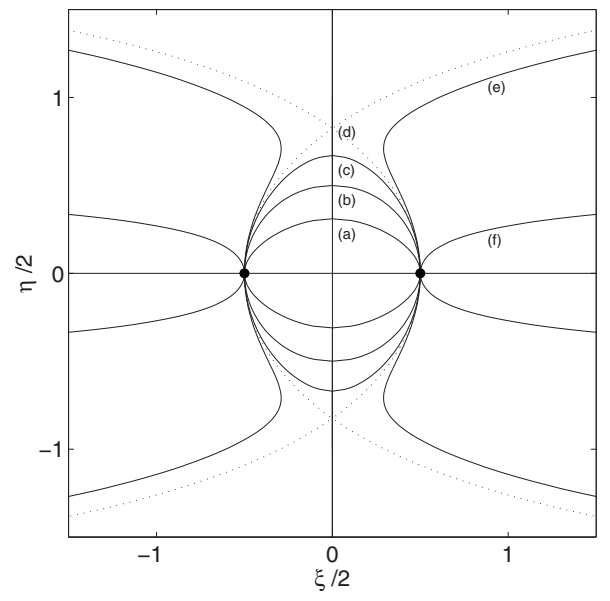

FIG. 2. Point-vortex trajectories for two vortices in linear shear: (a) $\mu=$ -0.796 , (b) $\mu=0$, (c) $\mu=0.103$, (d) $\mu=0.117$, (e) $\mu=0.135$, and (f) $\mu$ $=1.590$. The dotted line corresponds to the trajectories with $\mu=\mu_{c}$. The initial positions of the point vortices are indicated by the bullets.

$$
\xi^{2}+\eta^{2}=\xi_{0}^{2} \exp \left(\frac{\alpha \pi}{\gamma} \eta^{2}\right)
$$

where $\xi_{0}$ is the distance between the point vortices when they are horizontally aligned (i.e., $\eta=0$ ).

Figure 2 shows the trajectories of the point vortices for several values of $\mu$. We have confined ourselves to the solutions of Eq. (16) that have the initial point-vortex positions in common. The initial point-vortex positions are indicated by the bullets in Fig. 2. When no external shear is present $(\mu$ $=0$ ), the point vortices follow circular orbits due to vortexvortex interactions. For cooperative shear $(\mu<0)$, the point vortices move along closed orbits and their separation distance is minimum when the vortices are vertically aligned. For adverse shear of moderate strength, i.e., $0 \leq \mu<\mu_{c}$ with $\mu_{c}=1 / \pi e$ a critical value, the motion of the point vortices is also periodic, but now the separation distance is maximum when the point vortices are vertically aligned. For strong adverse shear, i.e., when $\mu>\mu_{c}$, the point vortices are separated indefinitely. The dotted lines in Fig. 2 represent the trajectories for the limiting case $\mu \downarrow \mu_{c}$. In fact, when $\mu=\mu_{c}$, the point vortices acquire a stationary state when they are vertically aligned. In this case the velocity of the shear exactly balances the velocity that each vortex induces in the other. Apart from this special case, it may be concluded from Fig. 2 that interactions between identical point vortices in linear shear results in either periodic or separative motion.

\section{CONTOUR DYNAMICS MODEL}

Despite being a helpful tool to model the main dynamical features of two vortices in linear shear, the applicability of the point-vortex model is limited to cases where the characteristic radii of finite-size vortices are much smaller than the distance between them. Obviously, this is not the case when the vortices merge, i.e., when the distance between the vortices is of the order of the vortex radius. In order to investigate the effect of distributed vorticity on the interaction of vortices in linear shear, the technique of contour dynamics is used. ${ }^{26}$ This technique allows us to calculate the evolution of vortices with distributed vorticity. We will focus on two different types of vortices: (1) the Rankine vortex, which is characterized by a core of uniform vorticity and zero vorticity in the outer region and which is relevant in $2 \mathrm{D}$ turbulent flows where the interaction with surrounding vortices leads to sharp-edged vortices, and (2) the Lamb vortex, which is characterized by a Gaussian vorticity distribution and closely resembles vortices as observed in the laboratory.

\section{A. Numerical procedure}

The two-vortex system in linear shear is governed by the material conservation of vorticity

$$
\frac{D \omega}{D t}=0
$$

where $D / D t$ is the material derivative for a two-dimensional scalar field advected by the local velocity $\boldsymbol{v}$, i.e., $D / D t$ $=\partial / \partial t+\boldsymbol{v} \cdot \nabla$, with $\nabla$ the two-dimensional gradient operator. The vorticity $\omega$ and the velocity $\boldsymbol{v}$ are related to the stream function $\psi$ by

$$
\omega=-\nabla^{2} \psi-\alpha
$$

and

$$
\boldsymbol{v}=-\boldsymbol{k} \times \nabla \psi+\boldsymbol{v}_{s},
$$

respectively, with $\boldsymbol{k}$ as the unit vector perpendicular to the plane of motion and $\boldsymbol{v}_{s}=(\alpha y, 0)$ as the background shear flow with strength $\alpha$. Note that the stream function $\psi$ is related to the velocity induced by the vortices only and not to the shear flow. Equations (17)-(19) are solved using the technique of contour dynamics. ${ }^{26,27}$ The essence of contour dynamics is that the distribution of a piecewise-uniform vorticity field is completely determined by the instantaneous positions of the contours separating the different regions of uniform vorticity. The contours are represented by a finite but adjustable number of nodes which are advected by the local velocity field Eq. (19), induced by the instantaneous vorticity distribution and the external linear shear. The velocity follows directly from the inversion of Poisson's Eq. (18) with the piecewiseuniform vorticity field as a source. Hence we have a closed dynamical system for fluid particles that lie on the contours. The number of nodes is allowed to change in time in response to possible deformations of the contours. The nodes are redistributed along the contours using cubic splines between the original nodes. In order to reduce the complexity of the contours and to be able to perform long-term time integrations, the technique of "contour surgery" is applied (see Ref. 27 for more details). Time integration was performed using a fourth-order Runge-Kutta method with a time step of $0.05\left(\omega_{m}\right)^{-1}$, with $\omega_{m}$ as the maximum vorticity of the vortex. The total area enclosed by the contours was conserved within $99 \%$.

\section{B. Rankine vortices}

The Rankine vortex is characterized by a core of uniform vorticity and an outer region of zero vorticity, i.e., 


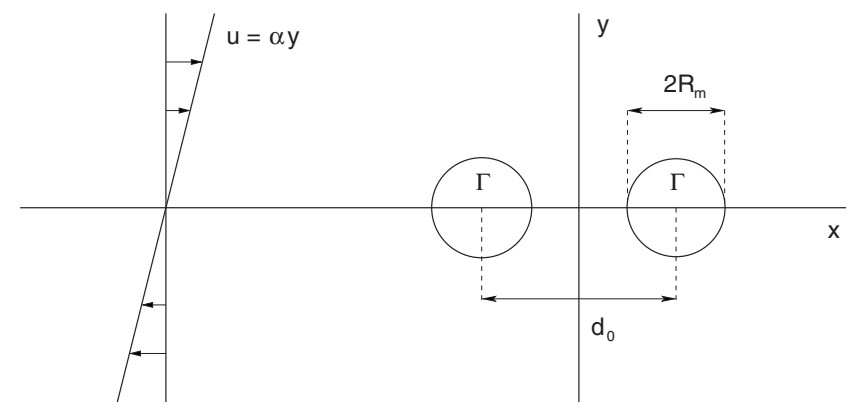

FIG. 3. Initial configuration of two Rankine vortices, each with circulation $\Gamma$ and radius $R_{m}$, in a linear shear flow with strength $\alpha$. The vortices are initially located an intercentroid distance $d_{0}$ apart.

$$
\begin{aligned}
& \omega=\frac{\Gamma}{\pi R_{m}^{2}} \text { for } 0 \leq r \leq R_{m}, \\
& \omega=0 \text { for } r>R_{m},
\end{aligned}
$$

with $\Gamma$ as the total circulation and $R_{m}$ as the vortex core radius. In general, $R_{m}$ is defined as the radius at which the azimuthal velocity is maximum, hence the subscript " $m$." Since there is only a single vorticity jump involved, the
Rankine vortex can be represented by a single contour. The initial configuration of the two Rankine vortices in linear shear is shown in Fig. 3. The Rankine vortices are initially aligned in the shear flow direction and located an intercentroid distance $d_{0}$ apart.

The time evolution of Rankine vortices in linear shear depends on two dimensionless parameters: $\alpha / \omega_{m}$ and $d_{0} / R_{m}$. The first parameter is the ratio of the shear strength $\alpha$ and the maximum vorticity of the vortex, $\omega_{m}=\Gamma /\left(\pi R_{m}^{2}\right)$. The second parameter is the ratio of the initial distance between the vortex centers, $d_{0}$, and the vortex radius $R_{m}$. In what follows, the parameters $\alpha / \omega_{m}$ and $d_{0} / R_{m}$ will be referred to simply as the shear strength and the initial separation distance, respectively, without explicit mention of the above normalization.

Figure 4 shows the characteristic flow regimes for fixed $d_{0} / R_{m}$ and different shear strengths $\alpha / \omega_{m}$ : (i) separative motion with elongation of the vortices [Fig. 4(a)], (ii) separative motion without elongation of the vortices [Fig. 4(b)], (iii) periodic motion [Figs. 4(c)-4(e)], and (iv) merger [Fig. 4(f)]. Many more simulations were performed, also for other values of $d_{0} / R_{m}$, but no other flow regimes were found (apart from a hybrid regime to be discussed below). The results are summarized in a regime diagram (see Fig. 5).

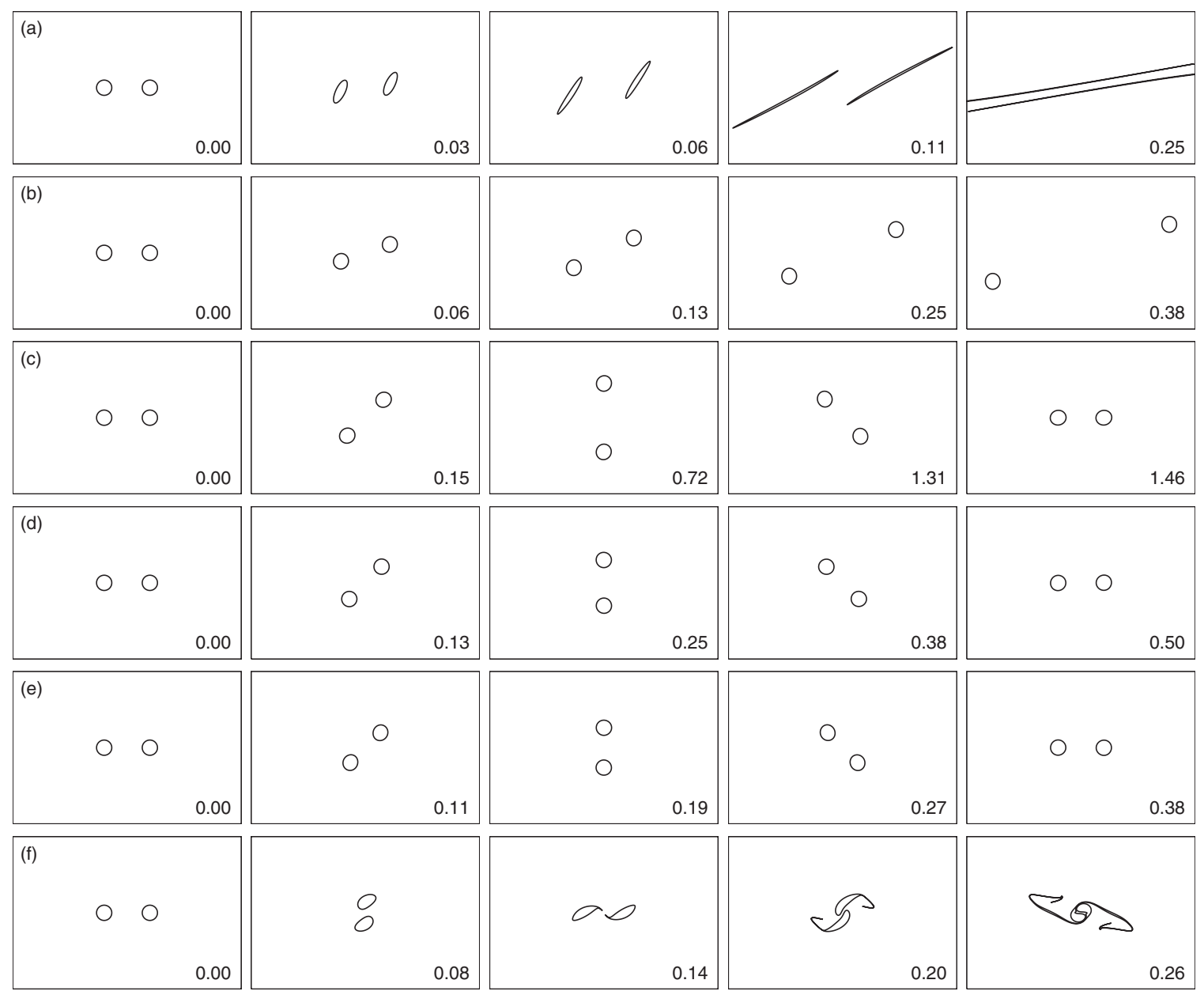

FIG. 4. Illustration of the different flow scenarios for two interacting Rankine vortices in linear shear. For each case, the initial separation distance $d_{0} / R_{m}$ $=6.0$, whereas the shear strength $\alpha / \omega_{m}$ is varied: (a) $\alpha / \omega_{m}=0.20$, separative motion with elongation; (b) $\alpha / \omega_{m}=0.05$, separative motion without elongation; (c) $\alpha / \omega_{m}=0.01$, periodic motion; (d) $\alpha / \omega_{m}=0$, periodic motion; (e) $\alpha / \omega_{m}=-0.01$, periodic motion; and (f) $\alpha / \omega_{m}=-0.15$, merger. Times are relative to the orbit period of two point vortices, each with circulation $\Gamma=\pi \omega_{m} R_{m}^{2}$, located an initial distance $d_{0}=6.0 R_{m}$ apart without external shear. 


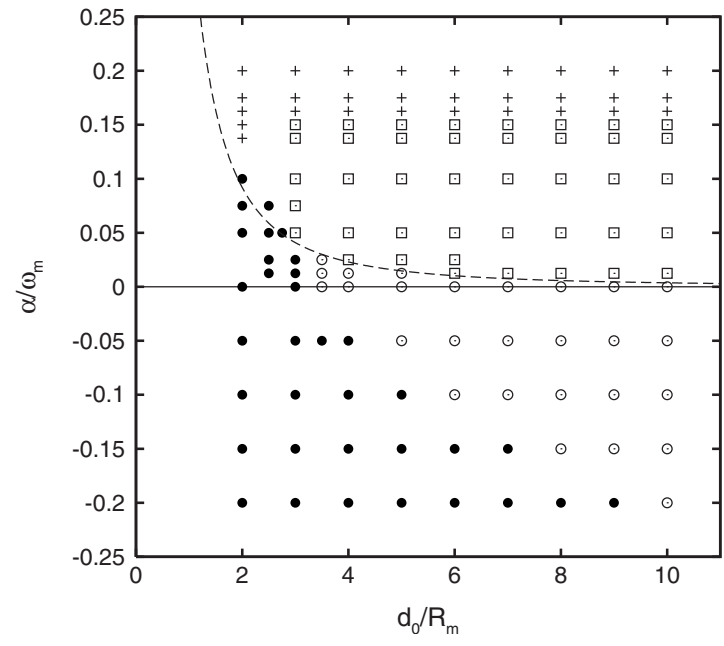

FIG. 5. Regime diagram for two Rankine vortices in linear shear, with $\alpha / \omega_{m}$ as the shear strength and $d_{0} / R_{m}$ as the initial separation distance. Separative motion with elongation is indicated by a plus $(+)$, separative motion without elongation by a square $(\square)$, periodic motion by a circle $(\bigcirc)$, and merger by a bullet ( ). The dashed line corresponds to the boundary between separative and periodic motion based on the point-vortex model.

For the case without shear $\left(\alpha / \omega_{m}=0\right)$, we may distinguish between two regimes. When the initial separation distance is larger than the critical separation distance, the vortex centers move along periodic trajectories [see Fig. 4(d)]. For initial separations smaller than the critical separation distance, the two vortices will merge. The critical separation distance $d_{0} / R_{m}$ is found to be $3.3 \pm 0.1$, which corresponds closely to the value stated in the literature. ${ }^{11,31,32}$

We now consider the effect of shear on the evolution of the Rankine vortices when the initial separation distance is relatively large, i.e., $d_{0} / R_{m}>3.3$. It is then found that for moderate adverse shear, the vortices still orbit periodically around each other, but now the trajectories are elongated in the $y$-direction [see Fig. 4(c)]. That is, the separation distance is effectively larger than that without shear, which was also the case for point vortices in adverse shear (see Sec. II). For larger adverse shear strengths, the trajectories of the vortex centers are no longer closed and the vortices move away from each other without being torn apart, as in Fig. 4(b). Upon further increasing the strength of the adverse shear, the vortices become strongly deformed and elongated [see Fig. 4(a)]. The centers of the vortices still follow open trajectories, as described above, but the strain induced by the shear is strong enough to tear each vortex apart.

When the vortices are exposed to cooperative shear and the initial separation distance is still relatively large, i.e., $d_{0} / R_{m}>3.3$, the periodic motion is preserved for moderate shear strengths, but the trajectories of the vortex centers are now flattened in the $y$-direction [see Fig. 4(e)]. That is, the separation distance is effectively smaller than that without shear, which was also the case for the point vortices (see Sec. II). For even stronger cooperative shear, the minimum separation distance is so small that the vortices merge [see Fig. 4(f)].

For relatively small initial separation distances, i.e., $d_{0} / R_{m}<3.3$, we observe from Fig. 5 that adverse shear in- hibits vortex merger when the shear strength is large enough. In this case, the vortices simply move away from each other by the action of the shear. When the shear is more adverse, the vortices are strongly deformed and each vortex is eventually torn apart. In contrast, cooperative shear brings the vortices effectively closer together and therefore promotes the merger process. It should be noted that for $d_{0} / R_{m}=2.0$, i.e., when the initial vortex cores touch, the distinction between the regimes of merger and separative motion with elongations was difficult to make for relatively large adverse shear $\left(\alpha / \omega_{m}>0.1\right)$. In these cases, the initial stage of the evolution was characterized by the formation of two elongated vortex cores which were intimately linked to one another, forming virtually a single elongated vortex, but later on, the centers of the original vortex cores drifted apart due to the action of the adverse shear. Despite the strong exchange of vorticity between the vortex cores, this hybrid evolution was assigned to the regime of separative motion with elongation.

Despite its simplicity and its possible shortcomings at relatively small separation distances, it is tempting to investigate whether the point-vortex model is able to predict some of the flow regimes for the case of two Rankine vortices in linear shear. A preliminary study on forced vortex merger by Waugh $^{31}$ has shown that such an approach has indeed potential. Some similarities between the evolution of Rankine vortices and point vortices have already been noted above. However, only a single parameter $\left(\mu=\alpha \xi_{o}^{2} / \gamma\right)$ is involved in the description of the motion of two point vortices in linear shear, whereas two parameters $\left(d_{0} / R_{m}\right.$ and $\left.\alpha / \omega_{m}\right)$ are required to parametrize the shear-induced evolution of two Rankine vortices. When the initial separation distance $\xi_{0}$ in the expression for $\mu$ [Eq. (1)] is replaced by the initial intercentroid distance $d_{0}$ between two Rankine vortices, and the circulation $\gamma$ in Eq. (1) is regarded as the circulation of a single Rankine vortex $\Gamma=\pi \omega_{m} R_{m}^{2}$, the parameter $\mu$ can be related to both $d_{0} / R_{m}$ and $\alpha / \omega_{m}$ as

$$
\mu=\frac{1}{\pi} \frac{\alpha}{\omega_{m}}\left(\frac{d_{0}}{R_{m}}\right)^{2} .
$$

Using the same procedure, the expression for the pointvortex trajectories [Eq. (16)] can be written in terms of $d_{0} / R_{m}$ and $\alpha / \omega_{m}$, i.e.,

$$
\left(\frac{\xi}{R_{m}}\right)^{2}+\left(\frac{\eta}{R_{m}}\right)^{2}=\left(\frac{d_{0}}{R_{m}}\right)^{2} \exp \left[\frac{\alpha}{\omega_{m}}\left(\frac{\eta}{R_{m}}\right)^{2}\right] .
$$

For point vortices in linear shear, the boundary between separative and periodic motion is determined by the critical parameter $\mu_{c}$. For Rankine vortices in linear shear, the same distinction can be made between separative motion and periodic motion. The relation between $\alpha / \omega_{m}$ and $d_{0} / R_{m}$ for $\mu$ $=\mu_{c}$ is given by

$$
\frac{\alpha}{\omega_{m}}=\pi \mu_{c}\left(\frac{d_{0}}{R_{m}}\right)^{-2} \text {. }
$$

This relation is indicated with the dashed line in the regime diagram displayed in Fig. 5. If the Rankine vortices would behave like point vortices, the dashed line would represent 
the boundary between periodic and separative motion for Rankine vortices. It is clear that the dashed line in Fig. 5 separates both regimes very well for values of $d_{0} / R_{m} \geqslant 3.0$. For smaller separation distances, the dashed line no longer represents the boundary between separative motion and periodic motion for Rankine vortices in linear shear. At these distances the point-vortex model is apparently no longer suitable due to the finite radii of the Rankine vortices.

It would be convenient to derive a simple criterion for the merger of two Rankine vortices in linear shear. When no shear is applied, the critical separation distance for the Rankine vortices is $d_{0} / R_{m}=3.3$. Although point vortices cannot merge, our numerical simulations demonstrated that up to the point of vortex merger, the point-vortex positions are in close agreement with the centroid positions of well-separated Rankine vortices. The separation distance between the point vortices may therefore serve as a good measure to predict merger. It is expected that Rankine vortices in shear will merge if the distance between the corresponding point vortices (which can be determined analytically) is always smaller than the critical value for merger of two Rankine vortices without shear. On the other hand, it is expected that the Rankine vortices will not merge if the distance between the corresponding point vortices is always larger than this critical value. Although these criteria are probably too strict, they may provide a sufficient condition for merger or no merger.

Because in the case of merger the distance between the centers of vorticity remains bounded, the following discussion focuses on situations with $\mu<\mu_{c}$, for which the interaction between point vortices results in periodic motion. For point vortices in adverse shear, the maximum separation distance is obtained when the point vortices are vertically aligned, i.e., $\xi=0$, whereas the minimum separation distance is obtained when the point vortices are horizontally aligned, i.e., $\eta=0$. In contrast, for point vortices in cooperative shear, the maximum and minimum separation distances correspond to the horizontal and vertical alignment of the vortices, respectively.

In Fig. 6 we show the same regime diagram as in Fig. 5. We now introduce two dashed lines. The vertical dashed line represents the case of two horizontally aligned point vortices which are located a critical distance $3.3 R_{m}$ apart, i.e., $\xi$ $=3.3 R_{m}$ and $\eta=0$. When the point vortices are embedded in cooperative shear, their intercentroid distance will never rise above the critical distance. Likewise, when the point vortices are embedded in adverse shear, their intercentroid distance will never fall below the critical distance. The other dashed line represents the case of two vertically aligned vortices which are located a critical distance $3.3 R_{m}$ apart, i.e., $\xi=0$ and $\eta=3.3 R_{m}$, where Eq. (23) has been used to derive the relationship between $\alpha / \omega_{m}$ and $d_{0} / R_{m}$. This case corresponds to the minimum separation distance when the point vortices are embedded in cooperative shear and to the maximum separation distance when the point vortices are embedded in adverse shear.

It is expected that in the region to the left of both lines, all interactions will result in merger, i.e., the distance between the vortices is always smaller than the critical value $3.3 R_{m}$. Likewise, in the region to the right of both lines, the

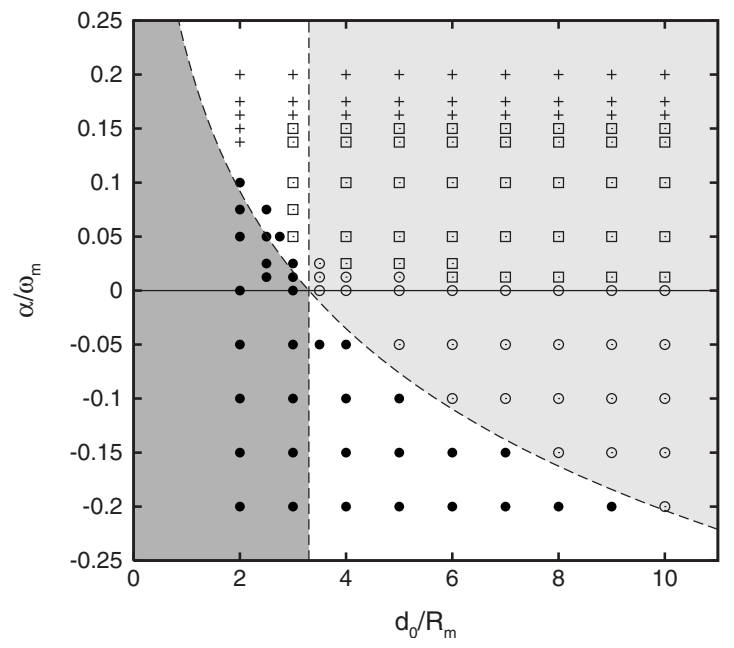

FIG. 6. Regime diagram for two Rankine vortices in linear shear. According to the point-vortex model, we may distinguish between merger (dark-shaded region) and no merger (light-shaded region). See the main text for details. The symbols have the same meaning as in Fig. 5.

distance between the two vortices is always larger than the critical distance and no merger is expected to occur. Both regions are highlighted in Fig. 6 with dark and light shading, respectively. It is clear that the prediction of merger or no merger is excellent. For the intermediate region, i.e., the unshaded regions, the regimes of merger and no merger coexist for vortices in adverse shear. In contrast, vortices in cooperative shear always merge in the intermediate region. As a result, the merger criterion for Rankine vortices in cooperative shear can be formulated more precisely by the requirement that the minimum separation distance between the vortices as predicted by the point-vortex model should be smaller than $3.3 R_{m}$.

We finally remark that for relatively large initial separation distances $\left(d_{0} / R_{m} \geq 3.0\right)$, the boundary between elongation and no elongation is given by $\alpha / \omega_{m}=0.156 \pm 0.006$, which can be verified from Figs. 5 and 6 . This value is in close agreement with the critical value derived analytically by Kida, ${ }^{21}$ who showed that a single Rankine vortex in a linear shear flow is torn apart when $\alpha / \omega_{m}>0.15$. For relatively small initial separation distances $\left(d_{0} / R_{m} \leqslant 3.0\right)$ up to the point of vortex merger, less ambient shear is required to break up the vortices since each vortex experiences additional strain induced by the other.

Summarizing, it is found that the evolution of two Rankine vortices in linear shear is much richer than that of two point vortices. Owing to the finite sizes of the vortices, the vortices merge when the initial separation distance is smaller than a critical value. The latter depends on both the sign and the strength of the linear shear. In addition, each vortex may be torn apart both by the straining action of the shear and the strain induced by the other vortex. Based on the point-vortex model, we derived a sufficient condition for merger and no merger of two Rankine vortices in linear shear. 


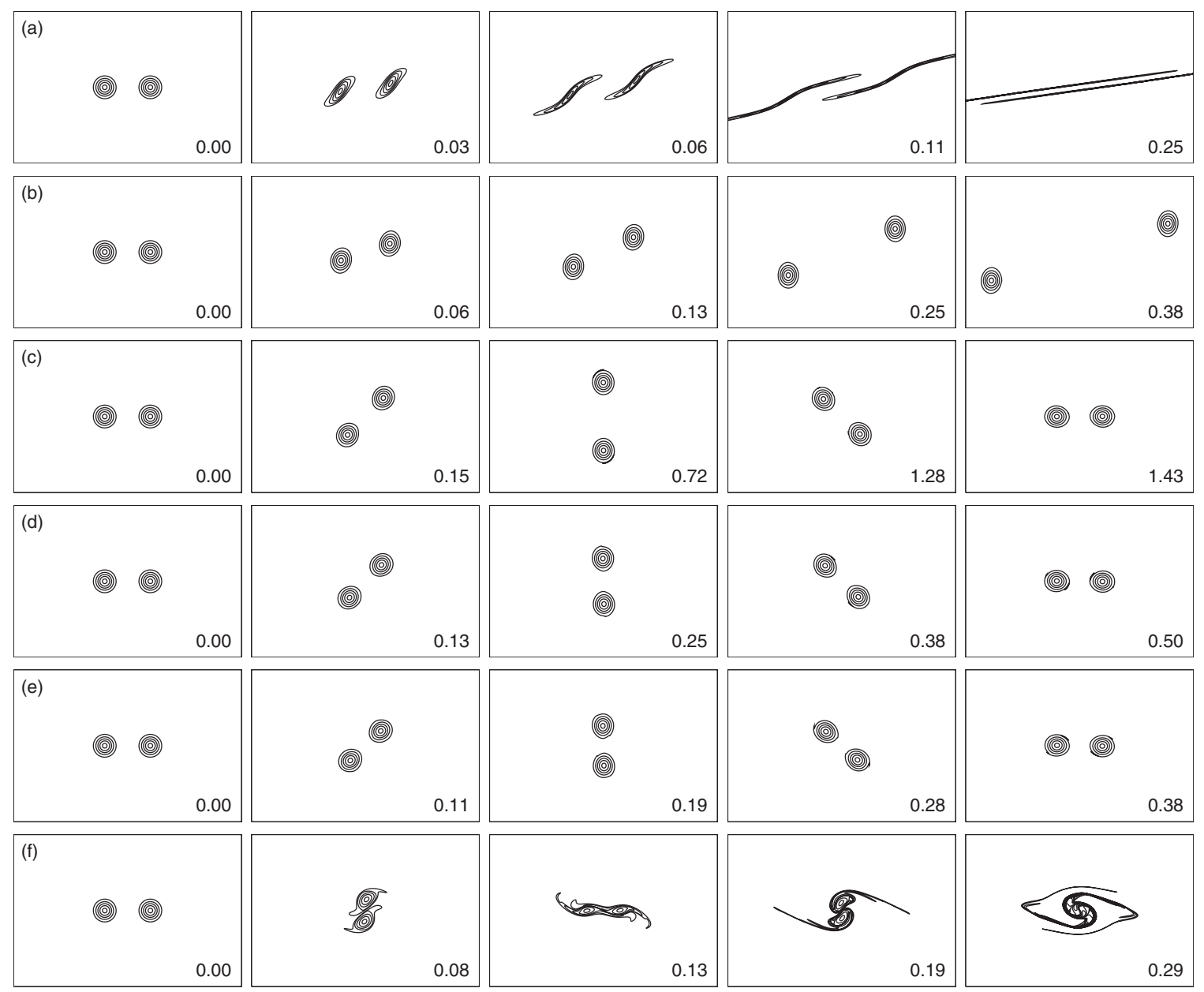

FIG. 7. Illustration of the different flow scenarios for two interacting Lamb vortices in linear shear. For each case, the initial separation distance $d_{0} / R_{m}$ $=5.35$, whereas the shear strength $\alpha / \omega_{m}$ is varied: (a) $\alpha / \omega_{m}=0.20$, separative motion with elongation; (b) $\alpha / \omega_{m}=0.05$, separative motion without elongation; (c) $\alpha / \omega_{m}=0.01$, periodic motion; (d) $\alpha / \omega_{m}=0$, periodic motion; (e) $\alpha / \omega_{m}=-0.01$, periodic motion; and (f) $\alpha / \omega_{m}=-0.15$, merger. For clarity, only the evolution of five selected contours is shown for each vortex, viz., at $\omega / \omega_{m}=0.95,0.70,0.47,0.28$, and 0.12 , respectively. Times are relative to the orbit period of two point vortices, each with circulation $\Gamma=\pi \omega_{m} R_{L}^{2}=\pi \omega_{m}\left(R_{m} / 1.12\right)^{2}$, located an initial distance $d_{0}=5.35 R_{m}=6.00 R_{L}$ apart without external shear.

\section{Lamb vortices}

In order to verify the above ideas for more realistic vortices, we consider the evolution of two Lamb vortices in linear shear. The Lamb vortex is characterized by a Gaussian vorticity distribution, i.e.,

$$
\omega=\frac{\Gamma}{\pi R_{L}^{2}} \exp \left(-r^{2} / R_{L}^{2}\right)
$$

which is often observed in the laboratory (see Sec. IV). Here, $R_{L}$ is a characteristic length scale which is related to the radius of maximum azimuthal velocity $R_{m}$ by $R_{m}=1.12 R_{L}$. We again apply the technique of contour dynamics to calculate the time evolution of the vortices in linear shear. For this, the continuous vorticity distribution of the Lamb vortex is discretized into 16 nested, uniform vorticity regions. Hence, each vortex is represented by 16 contours. This number of vortices is sufficient to resolve the "halo" of lowamplitude vorticity properly (see Ref. 19). The discretization is carried out in such a way that the discrete distribution of vorticity is closest to the continuous distribution in a leastsquares sense, under the constraint that the circulation of the two distributions is the same.

As for Rankine vortices, the flow is described by two dimensionless parameters: the initial separation distance $d_{0} / R_{m}$ and the shear strength $\alpha / \omega_{m}$, where the maximum vorticity $\omega_{m}$ is related to the circulation $\Gamma$ by $\omega_{m}=\Gamma /\left(\pi R_{L}^{2}\right)$. The vortices are initially aligned with the shear flow direction and located a distance $d_{0}$ apart.

Some characteristic results for various values of $d_{0} / R_{m}$ and $\alpha / \omega_{m}$ are shown in Fig. 7. Many more simulations were performed but no other regimes were found. The results are summarized in the regime diagram displayed in Fig. 8. The regime diagram for Lamb vortices is similar to that for Rankine vortices. Indeed, we observe the same four basic types of interaction: separative motion with and without elongation, periodic motion, and merger. The critical merger distance $d_{0} / R_{m}$ without shear is found to be $4.25 \pm 0.25$, whose value corresponds closely to the value 4.15 suggested in earlier studies. $^{13,15}$

Following the same procedure as before, we exploit the point-vortex model to predict some of the flow regimes for the case of two Lamb vortices in linear shear. Using Eq. (1) 


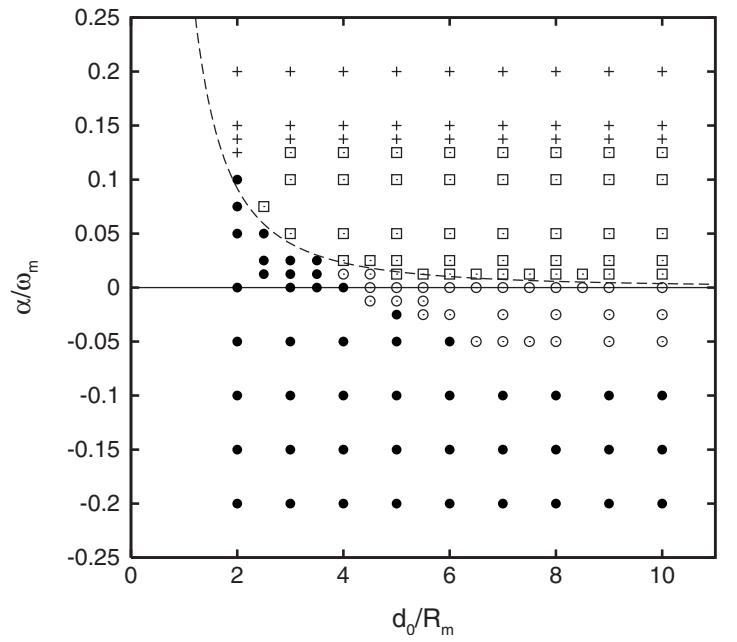

FIG. 8. Regime diagram for two Lamb vortices in linear shear. The dashed line corresponds to the boundary between separative and periodic motion based on the point-vortex model. The symbols have the same meaning as in Fig. 5.

and replacing $\xi_{0}$ with $d_{0}$ and $\gamma$ with $\Gamma=\pi \omega_{m} R_{L}^{2}$ $=\pi \omega_{m}\left(R_{m} / 1.12\right)^{2}$, we obtain similar expressions as for Eqs. (22)-(24).

The boundary between separative motion and periodic motion as derived from the point-vortex model is indicated in Fig. 8 by the dashed line. The line separates the corresponding flow regimes for Lamb vortices extremely well within the range of parameters investigated. We did not perform numerical simulations for smaller initial separation distances in order to avoid considerable overlap of the vorticity of each vortex (a situation not realistic in 2D turbulent flows).

We can derive a simple criterion for merger or no merger based on the point-vortex model as we did before for the system of two Rankine vortices. The Lamb vortices are expected to merge when the separation distance between the corresponding point vortices is always smaller than the critical merger distance associated with the Lamb vortices without shear $\left(d_{0} / R_{m}=4.15\right)$. On the other hand, no merger of the Lamb vortices is expected when the separation distance between the corresponding point vortices is always larger than this critical value. Using the same procedure as before, we may introduce two lines in the regime diagram, but now based on the critical merger distance for two Lamb vortices, which are expected to demarcate the regions of merger and no merger (see Fig. 9). To the left of both curves, the Lamb vortices are expected to merge, i.e., the distance between the corresponding point vortices never exceeds the critical value. To the right of both curves, the vortices are expected not to merge, i.e., the separation distance is never smaller than the critical value. In the intermediate region, both merger and no merger may occur. From Fig. 9 it is clear that the criterion for merger or no merger is very good. Also, for Lamb vortices in cooperative shear, we can formulate a more precise criterion for vortex merging by requiring that the minimum separation distance between the vortices as predicted by the point-vortex model should be smaller than $4.15 R_{m}$.

Concerning the regimes of elongation and no elongation,

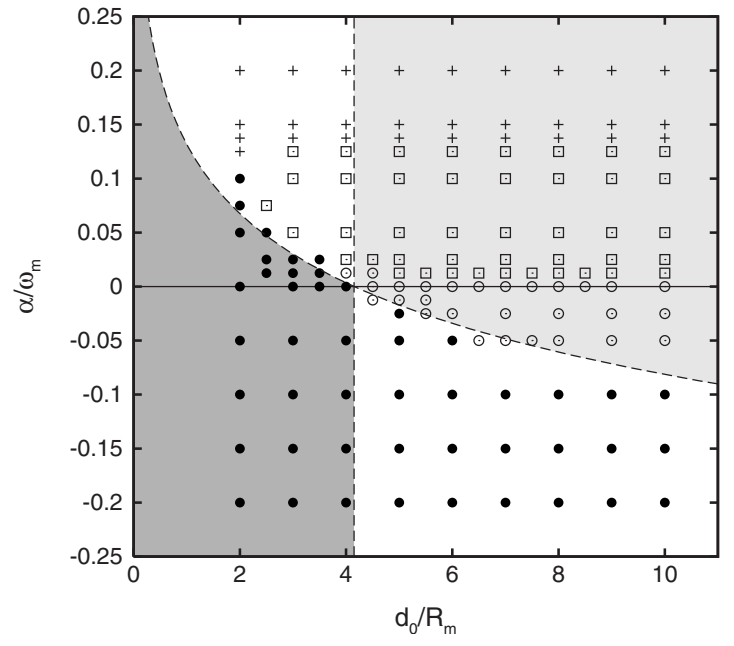

FIG. 9. Regime diagram for two Rankine vortices in linear shear. According to the point-vortex model, we may distinguish between merger (dark-shaded region) and no merger (light-shaded region). See the main text for details. The symbols have the same meaning as in Fig. 5.

we observe from either Figs. 8 and 9 that the corresponding transition occurs at $\alpha / \omega_{m}=0.131 \pm 0.006$. This value is in close agreement with numerical results by Mariotti et al. ${ }^{23}$ who found a critical value of $\alpha / \omega_{m}=0.134$ for a single Lamb vortex in linear shear.

A comparison between the regime diagrams for Rankine vortices and Lamb vortices reveals that Lamb vortices behave much in the same way as Rankine vortices when linear shear is applied. In both cases, the same four flow regimes are identified and the critical merger distances depend on both the sign and the strength of the shear flow. We also found a similar critical shear strength at which each vortex is torn apart. The shapes of the initial continuous vorticity profiles only affect the location of the boundaries of the flow regimes.

\section{LABORATORY EXPERIMENTS}

\section{A. Experimental procedure}

The laboratory experiments were performed in a rectangular tank with dimensions $200 \times 40 \times 30 \mathrm{~cm}$ (length $\times$ width $\times$ depth). The tank was mounted on a turntable with the tank center aligned with the axis of rotation. The tank was filled with water to a depth of approximately $20 \mathrm{~cm}$ and was set into motion with an angular velocity of $\Omega$ $=0.7 \mathrm{rad} \mathrm{s}^{-1}$ (which corresponds to a rotation period $T$ of 9.0 s). Before the vortices and the shear were created, the flow was allowed to adjust to solid-body rotation for at least half an hour. A schematic view of the experimental setup is depicted in Fig. 10. The Cartesian $(x, y)$ coordinate system is defined such that the $x$ - and $y$-axes are directed along the longer and shorter tank walls, respectively, with the origin located at the tank center.

Two cyclonic vortices were generated by locally siphoning fluid through two perforated tubes for typically one rotation period [see Fig. 10(a)]. The tubes were placed an initial distance $d_{0}$ apart on the long symmetry axis of the tank. 

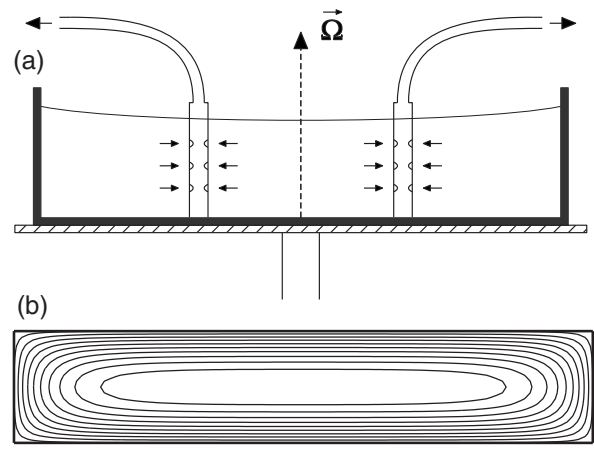

FIG. 10. Schematic representation of (a) the experimental setup (side view) and (b) the starting flow (without suction-induced vortices).

[Note that in Fig. 10(a) the distance between the tubes has been exaggerated for clarity.] During the forcing, a total amount of 1.01 of water was withdrawn. Owing to the presence of the Coriolis force, the radial motion induced by each sink was deflected in cyclonic direction. After the forcing was stopped and the tubes were removed from the tank, a well-defined swirling motion was established within a few rotation periods. The vortex Reynolds number was approximately 7500 .

The linear shear was created instantaneously afterward by a sudden change of the angular velocity of the tank $\Delta \Omega$ (typically $\pm 0.1 \mathrm{rad} \mathrm{s}^{-1}$ ), which resulted in a starting flow with uniform nonzero vorticity $-2 \Delta \Omega$ [see Fig. 10(b) and also Ref. 33]. This result can be easily understood from the conservation of absolute vorticity in a fixed frame of reference. When the fluid is in solid-body rotation, the absolute vorticity is given by $2 \Omega$. After the sudden change of the angular velocity of the tank, the vorticity associated with the rotation of the tank is $2 \Omega+2 \Delta \Omega$ so that the (relative) vorticity associated with the flow should be equal to $-2 \Delta \Omega$ in order to satisfy conservation of absolute vorticity. Depending on the sign of $\Delta \Omega$, the starting flow is either cyclonic (negative $\Delta \Omega$ ) or anticyclonic (positive $\Delta \Omega$ ). Since the initial flow within each vortex is cyclonically azimuthal, the cyclonic starting flow may be associated with cooperative shear, whereas the anticyclonic shear may be related to adverse shear. The initial flow conditions were controlled by variation of the separation distance of the tubes $d_{0}$ and the shear strength $\alpha=2 \Delta \Omega$. Both the suction rate and suction period were kept constant so that the initial vortex characteristics were the same for each experiment.

The subsequent flow evolution was visualized by injecting different colors of dye into the cores of the vortices just after the forcing was stopped. One vortex core was injected with "fluorescein" (green) and the other with "terasil brillant rosa" (red). Alternatively, the free surface was seeded with passive tracers (Optimage) with a mean particle diameter of $250 \mu \mathrm{m}$ in order to obtain quantitative information about the horizontal flow field. The flow was recorded from above with a corotating 12-bit charge-coupled device (CCD) camera with a resolution of $1024 \times 1024$ pixels and a frame rate of $15 \mathrm{~Hz}$. The technique of particle-tracking velocimetry was

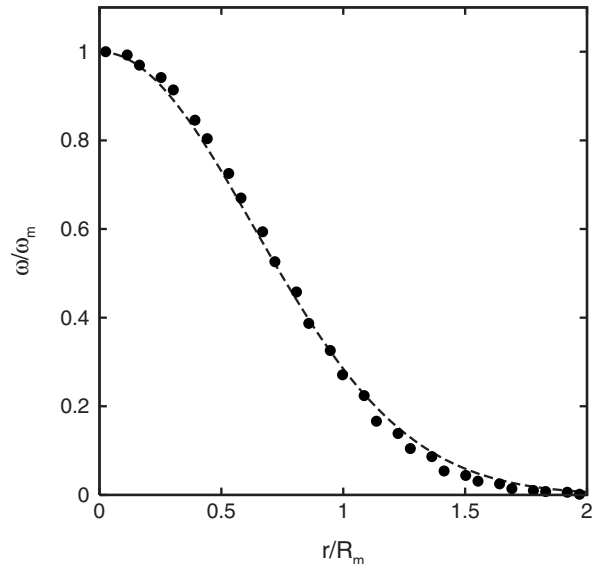

FIG. 11. Radial cross-sectional distribution of vorticity of a sink-induced cyclonic vortex (bullets) which is in close agreement with the Gaussian vorticity profile of a Lamb vortex (dashed line).

used to obtain the velocity of each tracer. In order to calculate the vorticity and the stream function, the measured velocities were interpolated on a rectangular grid of $50 \times 50$ mesh points.

\section{B. Experimental results}

Before we describe the evolution of the two suctioninduced vortices in linear shear, we discuss the characteristics of the vortices and the linear shear when they are generated in isolation.

Figure 11 shows the radial distribution of vorticity for a single vortex generated by the suction technique without ambient shear at six rotation periods after the forcing was stopped. The measured profile (indicated by the bullets) closely agrees with the Gaussian vorticity profile of the Lamb vortex. The corresponding vortex core radius $R_{m}$ and maximum vorticity $\omega_{m}$ were taken as the initial conditions for all experiments. At earlier times these quantities could not be determined accurately due to transient disturbances induced by the lifting of the tubes. As for the shear flow, contour plots of the stream function (not shown) reveal that the topology of the starting flow (without suction-induced vortices) is very similar to that shown schematically in Fig. 10(b). Figure 12 displays three cross-sectional distributions of the streamwise velocity component at $x=-\frac{1}{2} B, 0$, and $\frac{1}{2} B$, with $B$ the width of the tank. The shear is close to linear in the central part of the flow domain, except near the tank walls where the velocity is reduced to zero within a thin boundary layer. Moreover, it is evident from Fig. 12 that the corresponding shear strengths $\alpha$, as obtained from the slopes of the cross-sectional distributions of velocity, are in close agreement with the theoretical value $2 \Delta \Omega$. The linearity of the shear flow was preserved for at least 20 rotation periods. After that, the linear shear was gradually destroyed by flow separation at the lateral tank walls and the subsequent change of the topology of the flow into an array of alternately cyclonic and anticyclonic cells. These cells are typical for spin up and spin down in rectangular containers. ${ }^{33}$ We also note that both the maximum vorticity of each vortex and the magnitude of the shear strength decrease exponentially in time 


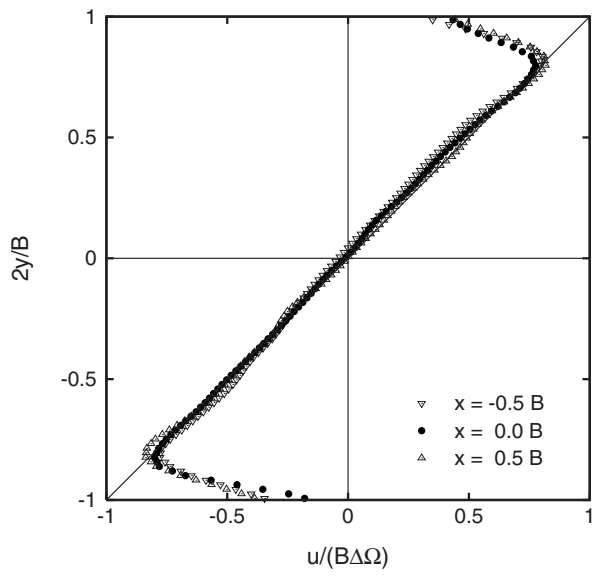

FIG. 12. Cross-sectional distributions of streamwise shear velocity at $x=$ $-\frac{1}{2} B, 0$, and $\frac{1}{2} B$, with $B$ as the width of the tank. The solid line corresponds to the theoretical velocity profile of the starting flow with $\Delta \Omega$ $=0.05 \mathrm{rad} \mathrm{s}^{-1}$.

due to the presence of Ekman boundary layers at the bottom of the rotating tank. Their ratio, however, remains constant as long as linear Ekman damping is the only source of decay.

Figure 13 shows an example evolution of two dyevisualized vortices in cooperative shear for $d_{0} / R_{m}=8.8$ and $\alpha / \omega_{m}=-0.12$. Times are indicated in the lower-right corner of each panel and are relative to the time at which the linear shear was applied, i.e., the time at which the rotation speed of the turntable was suddenly changed. Each panel corresponds to a free-surface area of $40 \times 20 \mathrm{~cm}^{2}$. The white dots, as visible in each panel, are remnants of passive tracers which were used to quantify the shear flow characteristics in a previous experiment. The initial distance between the vor- tices is significantly larger than the critical separation distance $d_{0} / R_{m}=4.15$ associated with the merger of two Lamb vortices without external shear. As we will see, however, cooperative shear promotes the merger of vortices despite the fact that their initial separation distance is significantly larger than $d_{0} / R_{m}=4.15$.

During the initial stage of the evolution, the vortices orbit around each other in a cyclonic sense. At $t=3.0 T$ (first panel of Fig. 13), the vortices have already completed half an orbit period. As is evident from the next panel $(t=4.5 T)$, the vortices are advected toward each other by the shear and hence their separation distance decreases considerably. At the same time, each vortex acquires a pearlike shape and forms two spiral arms. When the vortices have reached their closest distance, the vortices start to exchange fluid through one of their spiral arms $(t=6.0 T)$. The shedding of filaments and the subsequent exchange of fluid are usually indicative of the onset of vortex merger. Owing to the action of the shear, the separation distance between the vortices temporarily increases again until the vortices are aligned with the flow direction of the shear $(t=7.5 T)$. Note, however, that at this stage the separation distance is smaller than half an orbit period before (compare the panels for $t=3.0 T$ and $t=7.5 T$ ). From this stage, the evolution as described above repeats itself, but now the formation of filaments and the exchange of fluid is more vigorous than before because the vortices are closer together. At $t=12.0 T$, the vortices are again horizontally aligned, but now their distance has become so small that in the next stage, the vortices finally merge. The evolution of the laboratory vortices is in close agreement with the com-
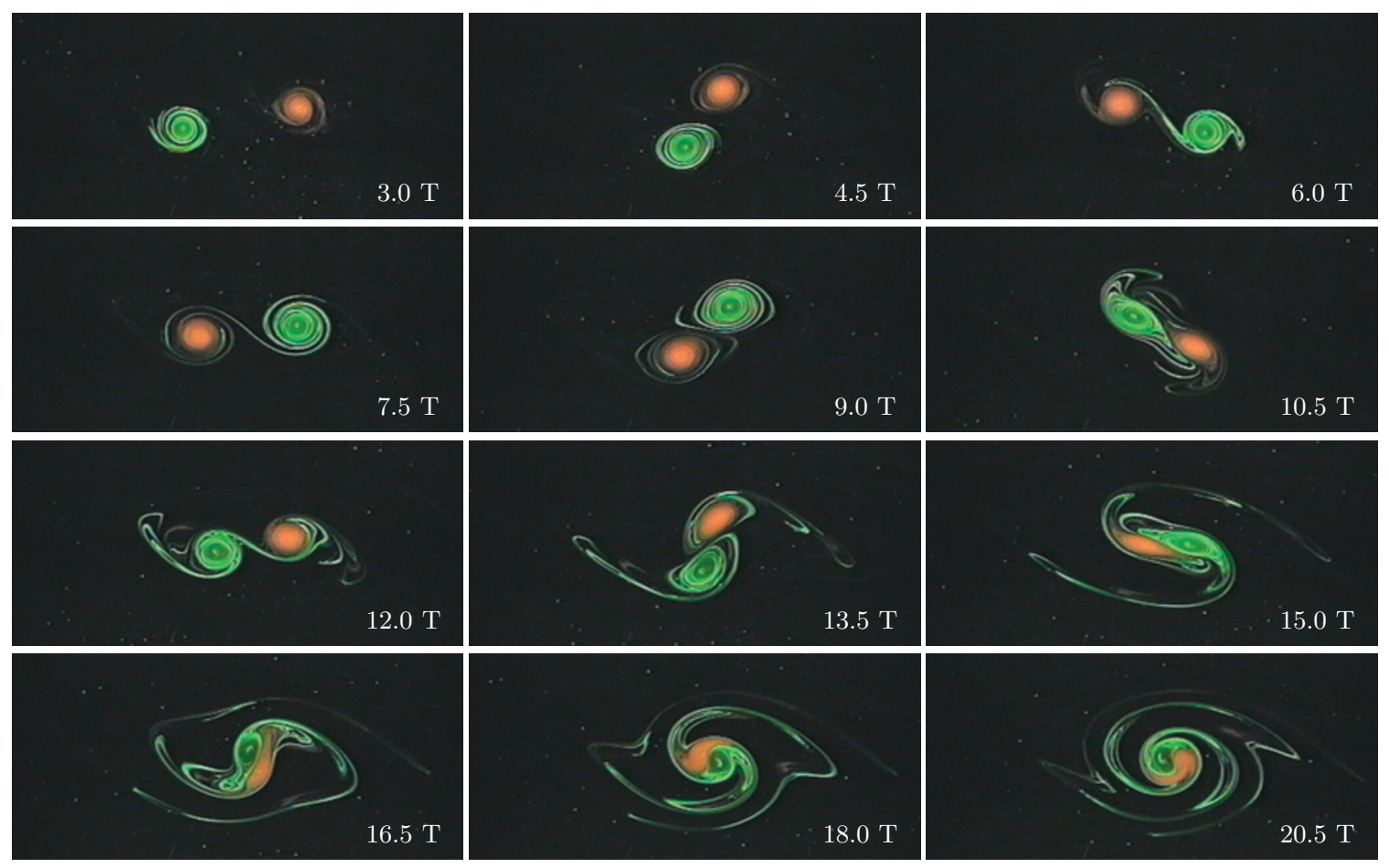

FIG. 13. (Color) Dye visualization of two laboratory vortices in cooperative shear with $d_{0} / R_{m}=8.8$ and $\alpha / \omega_{m}=-0.12$ at successive times at which the shear flow was imposed. Each panel corresponds to a free-surface area of $40 \times 20 \mathrm{~cm}^{2}$. Experimental parameters: $d_{0}=30 \mathrm{~cm}$ and $\alpha=-0.13 \mathrm{~s}$. 

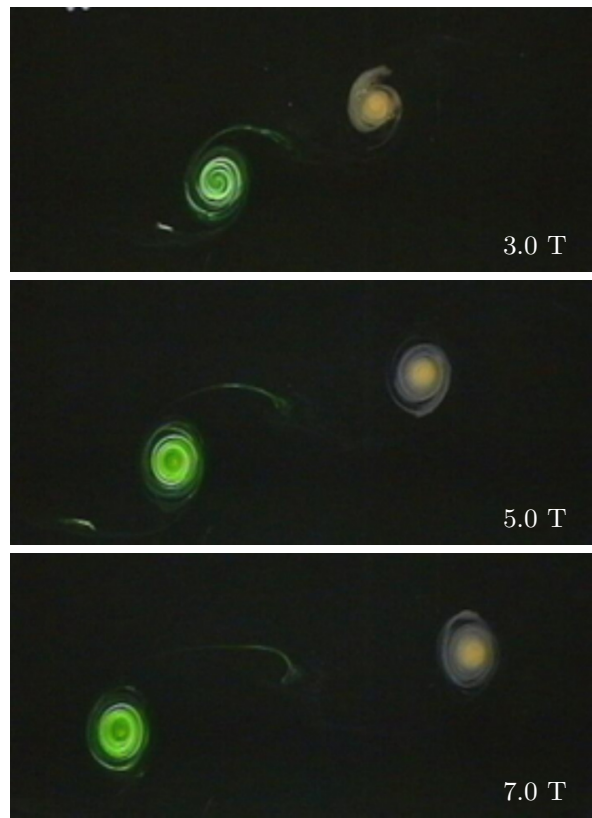

FIG. 14. (Color online) Dye visualization of two laboratory vortices in adverse shear with $d_{0} / R_{m}=4.4$ and $\alpha / \omega_{m}=0.05$ at successive times at which the shear flow was imposed. Experimental parameters: $d_{0}=15 \mathrm{~cm}$ and $\alpha$ $=0.06 \mathrm{~s}^{-1}$.

puted evolution of two Lamb vortices with $d_{0} / R_{m}=8.8$ and $\alpha / \omega_{m}=-0.12$ (not shown) and qualitatively similar to that shown in Fig. 7(f).

In Fig. 14 we show an example evolution of two dyevisualized vortices in adverse shear for $d_{0} / R_{m}=4.4$ and $\alpha / \omega_{m}=0.05$. Again, each panel corresponds to a free-surface area of $40 \times 20 \mathrm{~cm}^{2}$. As evident from the subsequent panels, the vortices quickly move away from each other without being torn apart. Each vortex acquires an elliptical shape with its major axis virtually perpendicular to the shear flow direction. Close inspection of the flow evolution reveals that each vortex is subjected to small-amplitude shape oscillations. In addition, one of the vortices demonstrates the formation of dye filaments which are characteristic for the process of vortex stripping. (The other vortex does not show dye filaments because unintentionally a smaller amount of dye was released by hand). All these observations are typical for single vortices in adverse shear ${ }^{21-25}$ and are in close agreement with the computed evolution shown in Fig. 7(b). [The apparent absence of the vortex filaments in Fig. 7(b) is due to the fact that only five contours are displayed for clarity].

For each dye-visualized experiment, the flow evolution was monitored for 20 rotation periods. After that, the interaction process was assigned to one of the flow regimes predicted in Sec. III C, which resulted in the regime diagram depicted in Fig. 15. Each experiment was performed at least twice to check whether it would reproduce. Obviously, the range of experimental parameters was limited owing to the finite dimensions of the tank. Indeed, for some initial configurations in adverse shear-especially those that were expected to lead to periodic motion or separative motion with elongation-the vortices were advected into the boundary layer along the longer tank walls or were drifted toward the

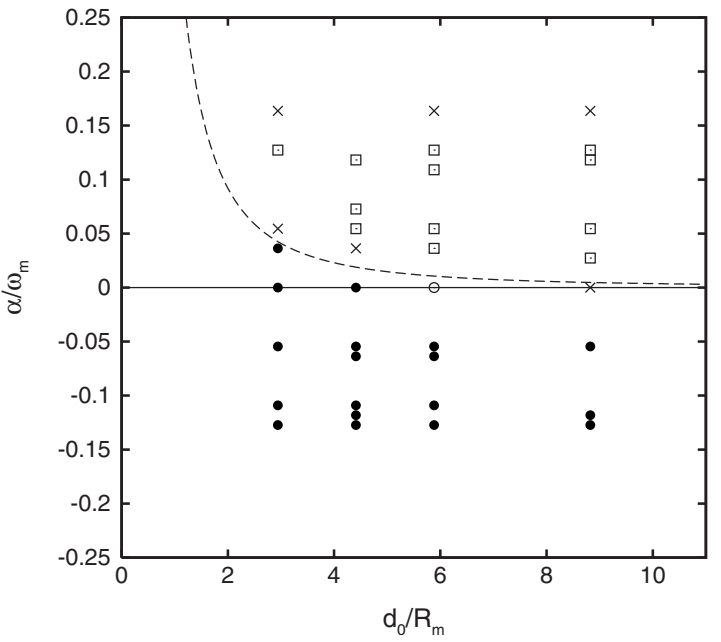

FIG. 15. Regime diagram for two laboratory vortices in linear shear. The dashed line corresponds to the boundary between separative and periodic motion based on the point-vortex model. Owing to the finite dimensions of the tank, some flow evolutions could not be assigned to any of the flow regimes. These cases are indicated with a cross $(\times)$. The other symbols have the same meaning as in Fig. 5.

shorter tank walls where a linear shear was no longer present. These cases were not assigned to any of the flow regimes and are indicated by a cross in the regime diagram of Fig. 15.

The regime diagram in Fig. 15 is very similar to that derived numerically for Lamb vortices. Also plotted in Fig. 15 is the boundary between separative motion and periodic motion as predicted by the point-vortex model. As far as can be judged from the present number of data points, the predicted boundary agrees well with the laboratory experiments. Figure 16 shows the same experimental results but now with the criterion for merger or no merger based on the pointvortex model and the critical merger distance for two Lamb vortices without shear. The criterion is generally satisfied,

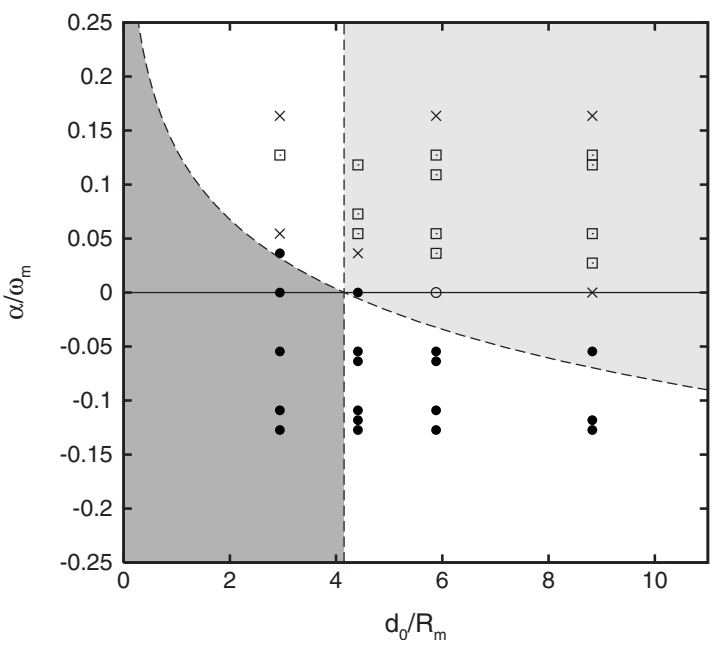

FIG. 16. Regime diagram for two laboratory vortices in linear shear. According to the point-vortex model we may distinguish between merger (dark-shaded region) and no merger (light-shaded region). See the main text for details. Flow evolutions that could not be assigned to any of the flow regimes are indicated with a cross $(\times)$. The other symbols have the same meaning as in Fig. 5. 
although for two cases we observe vortex merger where the point-vortex model predicts no merger. These small differences may be attributed to the radial expansion of the vortices owing to horizontal diffusion and nonlinear Ekman effects, both of which promote vortex merger. Moreover, vortex merger is promoted by the parabolic free surface of the fluid, which effectively moves the cyclonic vortices toward the center of the tank due to topography vorticity production. ${ }^{13}$ In this respect, the locations of the boundaries of the observed flow regimes may be sensitive to the time at which the flow evolution was evaluated. Note, however, that the initial conditions for the laboratory vortices were taken at the time at which the Rossby number $\mathrm{Ro}=\omega_{m} /(2 \Omega)=0.8$, whose value decreases close to exponentially, so that the radial expansion due to nonlinear Ekman effects is only effective during the initial stage of the evolution. As far as horizontal diffusion is concerned, one should keep in mind that vortex merger itself is a nonlinear process which evolves on a convective time scale (typically one or two orbit periods). That is, the motion of the vortex centroids toward each other is a process which is virtually independent of viscosity (see, for example, Ref. 4). Vortex interactions that do not lead to merger in the inviscid limit may still merge when viscous dissipation is present. In that case, vortex merger is preceded by a diffusive stage, typically of the order of the diffusive time scale, in which the vortex cores grow until the inviscid critical separation distance is reached at which the vortices merge. In the current laboratory experiments, the vortex flow was evaluated within a time period much smaller than the diffusive time scale. That is, considering the characteristic diffusive time scale with respect to the rotation period, $T_{d} / T \sim 10^{2}$ with $T_{d}=R_{m}^{2} / \nu$, and the fact that the flow evolution was evaluated at $t=20 T$, the effect of horizontal diffusion is only of secondary importance. Despite the small differences, the experimental data convincingly illustrate the different behavior of vortices in adverse and cooperative shear.

\section{SUMMARY AND CONCLUSIONS}

The interaction of two identical vortices in a linear shear has been investigated both numerically and experimentally. As a first approach, we considered a system of two interacting point vortices in linear shear in order to understand the key dynamical aspects of the flow evolution. For this we closely followed the analysis by Kimura and Hasimoto. ${ }^{30}$ Essentially, two types of interaction were identified: separative motion and periodic motion. The flow evolution could be characterized by a single dimensionless parameter which is proportional to the shear strength. For cooperative shear, the point vortices always move along closed orbits which are elongated in the direction of the shear flow. Likewise, for adverse shear of moderate strength, the point vortices move along closed orbits, but the orbits are elongated in the direction perpendicular to that of the shear flow. For strong adverse shear, the point vortices are separated indefinitely.

As a second approach, we applied the technique of contour dynamics to investigate the effect of distributed vorticity on the interaction of vortices in linear shear. Two different types of vortices were considered: (1) the Rankine vortex with a core of uniform vorticity and zero vorticity in the outer region and (2) the Lamb vortex with a Gaussian vorticity distribution. Numerical calculations revealed that their shear-induced evolution is much richer than that of two point vortices. The following flow regimes were identified: (i) separative motion with elongation of the vortices, (ii) separative motion without elongation of the vortices, (iii) periodic motion, and (iv) merger. Owing to the distribution of vorticity, the vortices merge when their initial separation distance is smaller than some critical distance. The critical separation distance depends strongly on both the sign and the strength of the linear shear and, to a lesser extent, on the initial vorticity distribution. Based on the sign dependence of the critical merger distance, we conclude that cooperative shear promotes merger, whereas adverse shear inhibits merger. At a critical adverse shear strength, whose value depends on the initial vorticity distribution, the vortices are irreversibly torn apart.

The numerical results for Lamb vortices were in close agreement with rotating-tank experiments in which the vortices were created by the suction method, whereas the linear shear was created by a sudden increase in the angular velocity of the tank. A number of experiments were performed with different values for the shear strength and the intercentroid distance. The subsequent flow evolution was visualized by injecting different colors of dye into the cores of the initial vortices, whereas particle-tracking techniques provide quantitative information about the horizontal flow field. Small deviations were observed due to horizontal diffusion, nonlinear Ekman decay and topography induced motion.

Based on the point-vortex model, a sufficient condition for the merger of two distributed vortices in linear shear was derived. For distributed vortices in cooperative shear, a stricter criterion for vortex merging was formulated. Moreover, for relatively well-separated distributed vortices, the point-vortex model predicts the boundary between separative and periodic motion remarkably well. In the framework of freely decaying 2D turbulence, the merger criteria derived in this study are valuable to improve punctuated Hamiltonian models. ${ }^{13,34}$ In these models, spatially extended vortex structures are represented by point vortices and simple transformation rules are used to replace two vortices by a single one. Obviously, in future works, the merger criteria should then be extended in order to model the interaction of unequal vortices in linear shear flow correctly.

\section{ACKNOWLEDGMENTS}

We are grateful to Professor David Dritschel for providing the source code of the contour dynamics algorithm. One of the authors (R.R.T.) gratefully acknowledges financial support by the Royal Netherlands Academy of Arts and Sciences (KNAW).

${ }^{1}$ J. C. Mcwilliams, "The emergence of isolated coherent vortices in turbulent flow," J. Fluid Mech. 146, 21 (1984).

${ }^{2}$ B. Legras, P. Santangelo, and R. Benzi, "High-resolution numerical experiments for forced two-dimensional turbulence," Europhys. Lett. 3, 811 (1988). 
${ }^{3}$ M. V. Melander, N. J. Zabusky, and J. C. McWilliams, "Symmetric vortex merger in two dimensions: Causes and conditions," J. Fluid Mech. 195, 303 (1988).

${ }^{4}$ C. Cerretelli and C. H. K. Williamson, "The physical mechanism for vortex merging," J. Fluid Mech. 475, 41 (2003).

${ }^{5}$ M. J. Huang, "The physical mechanism of symmetric vortex merger: A new viewpoint," Phys. Fluids 17, 074105 (2005).

${ }^{6}$ O. U. Velasco Fuentes, "Vortex filamentation: Its onset and its role on axisymmetrization and merger," Dyn. Atmos. Oceans 40, 23 (2005).

${ }^{7}$ L. K. Brandt and K. K. Nomura, "The physics of vortex merger: Further insight," Phys. Fluids 18, 051701 (2006).

${ }^{8}$ L. K. Brandt and K. K. Nomura, "The physics of vortex merger and the effects of ambient stable stratification," J. Fluid Mech. 592, 413 (2007).

${ }^{9}$ V. J. Rosso, "Convective merging of vortex cores in lift-generated wakes," J. Aircr. 14, 283 (1977).

${ }^{10}$ P. G. Saffman and R. Szeto, "Equilibrium shapes of a pair of equal uniform vortices," Phys. Fluids 23, 2339 (1980).

${ }^{11}$ E. A. Overman and N. J. Zabusky, "Evolution and merger of isolated vortex structures," Phys. Fluids 25, 1297 (1982).

${ }^{12}$ R. W. Griffiths and E. J. Hopfinger, "Coalescing of geostrophic vortices," J. Fluid Mech. 178, 73 (1987).

${ }^{13}$ G. F. Carnevale, P. Cavazza, P. Orlandi, and R. Purini, "An explanation for anomalous vortex merger in rotating-tank experiments," Phys. Fluids A 3, 1411 (1991).

${ }^{14}$ U. Ehrenstein and M. Rossi, "Equilibria of corotating nonuniform vortices," Phys. Fluids 11, 3416 (1999).

${ }^{15}$ P. Meunier, U. Ehrenstein, T. Leweke, and M. Rossi, "A merging criterion for two-dimensional co-rotating vortices," Phys. Fluids 14, 2757 (2002).

${ }^{16}$ M. V. Melander, N. J. Zabusky, and J. C. McWilliams, "Asymmetric vortex merger in two dimensions: Which vortex is 'victorious'?," Phys. Fluids 30, 2610 (1987).

${ }^{17}$ D. G. Dritschel and D. W. Waugh, "Quantification of the inelastic interaction of unequal vortices in two-dimensional vortex dynamics," Phys. Fluids A 4, 1737 (1992).

${ }^{18}$ T. B. Mitchell and C. F. Driscoll, "Electron vortex orbits and merger," Phys. Fluids 8, 1828 (1996).

${ }^{19}$ R. R. Trieling, O. U. Velasco Fuentes, and G. J. F. van Heijst, "Interaction of two unequal corotating vortices," Phys. Fluids 17, 087103 (2005).

${ }^{20}$ L. K. Brandt and K. K. Nomura, "Characterization of the interactions of two unequal co-rotating vortices," J. Fluid Mech. 646, 233 (2010).

${ }^{21}$ S. Kida, "Motion of an elliptic vortex in a uniform shear flow," J. Phys. Soc. Jpn. 50, 3517 (1981).

${ }^{22}$ B. Legras and D. G. Dritschel, "Vortex stripping and the generation of high vorticity gradients in two-dimensional flows," Appl. Sci. Res. 51, 445 (1993)

${ }^{23}$ A. Mariotti, B. Legras, and D. G. Dritschel, "Vortex stripping and the erosion of coherent structures in two-dimensional flows," Phys. Fluids 6, 3954 (1994).

${ }^{24}$ O. Paireau, P. Tabeling, and B. Legras, "A vortex subjected to a shear: An experimental study," J. Fluid Mech. 351, 1 (1997).

${ }^{25}$ R. R. Trieling, A. H. Linssen, and G. J. F. van Heijst, "Monopolar vortices in an irrotational annular shear flow," J. Fluid Mech. 360, 273 (1998).

${ }^{26}$ N. J. Zabusky, M. H. Hughes, and K. V. Roberts, "Contour dynamics for the Euler equations in two dimensions," J. Comput. Phys. 30, 96 (1979).

${ }^{27}$ D. G. Dritschel, "Contour dynamics and contour surgery: Numerical algorithms for extended, high-resolution modeling of vortex dynamics in twodimensional, inviscid, incompressible flows," Comput. Phys. Rep. 10, 77 (1989).

${ }^{28}$ R. C. Kloosterziel and G. J. F. van Heijst, "The evolution of stable barotropic vortices in a rotating free-surface fluid," J. Fluid Mech. 239, 607 (1992).

${ }^{29}$ R. Kunnen, R. R. Trieling, and G. J. F. van Heijst, "Vortices in timeperiodic shear," Theor. Comput. Fluid Dyn. 24, 315 (2010).

${ }^{30}$ Y. Kimura and H. Hasimoto, "Motion of two identical point vortices in a simple shear flow," J. Phys. Soc. Jpn. 54, 4069 (1985).

${ }^{31}$ D. W. Waugh, "Forced vortex merger," Woods Hole Oceanographic Institution Technical Report No. WHOI-92-16, 1991, pp. 250-271.

${ }^{32}$ E. J. Hopfinger and G. J. F. van Heijst, "Vortices in rotating fluids," Annu. Rev. Fluid Mech. 25, 241 (1993).

${ }^{33}$ G. J. F. van Heijst, P. A. Davies, and R. G. Davis, "Spin-up in a rectangular container," Phys. Fluids A 2, 150 (1990).

${ }^{34}$ R. Benzi, M. Colella, M. Briscolini, and P. Santangelo, "A simple point vortex model for two-dimensional decaying turbulence," Phys. Fluids A 4, 1036 (1992). 